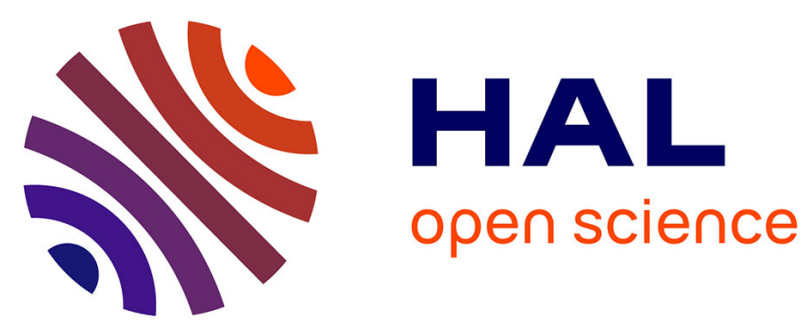

\title{
Pattern Rejection Strategies for the Design of Self-Paced EEG-based Brain-Computer Interfaces
}

\author{
Fabien Lotte, Harold Mouchère, Anatole Lécuyer
}

\section{To cite this version:}

Fabien Lotte, Harold Mouchère, Anatole Lécuyer. Pattern Rejection Strategies for the Design of SelfPaced EEG-based Brain-Computer Interfaces. International Conference on Pattern Recognition, Dec 2008, Tampa, United States. inria-00310878

\section{HAL Id: inria-00310878 \\ https://hal.inria.fr/inria-00310878}

Submitted on 11 Aug 2008

HAL is a multi-disciplinary open access archive for the deposit and dissemination of scientific research documents, whether they are published or not. The documents may come from teaching and research institutions in France or abroad, or from public or private research centers.
L'archive ouverte pluridisciplinaire HAL, est destinée au dépôt et à la diffusion de documents scientifiques de niveau recherche, publiés ou non, émanant des établissements d'enseignement et de recherche français ou étrangers, des laboratoires publics ou privés. 


\title{
Pattern Rejection Strategies for the Design of Self-Paced EEG-based Brain-Computer Interfaces
}

\author{
Fabien Lotte ${ }^{1,2,3}$, Harold Mouchère ${ }^{1,2}$, Anatole Lécuyer ${ }^{1,3}$ \\ IRISA $^{1} /$ INSA $^{2} /$ INRIA $^{3}$ \\ Avenue du général Leclerc, 35042 Rennes, France \\ firstname.lastname@irisa.fr
}

\begin{abstract}
This paper deals with pattern rejection strategies for self-paced Brain-Computer Interfaces (BCI). First, it introduces two pattern rejection strategies not used yet for self-paced BCI design: 1) the rejection class $(R C)$ strategy and 2) thresholds on reliability functions (TRF) based on the automatic multiple-threshold learning algorithm. Second, it compares several rejection strategies using several classifiers, on motor imagery data, in order to identify their most desirable properties. Results showed that nonlinear classifiers led to the most efficient self-paced BCI. Concerning the reject option, $R C$ outperformed a specialized reject classifier which outperformed TRF. Overall, the best results were obtained using the $R C$ reject option and non-linear classifiers such as a Gaussian support vector machine, a fuzzy inference system or a radial basis function network.
\end{abstract}

\section{Introduction}

Brain-Computer Interfaces (BCI) based on ElectroEncephaloGraphy (EEG) enable users to send commands to computers only by means of brain activity, measured using EEG [12]. To use a BCI, the user has to produce brain activity patterns which will be identified by the system [3] and translated into commands. Due to the noisy and unstable nature of EEG signals, the design of a $\mathrm{BCI}$ is known to be a challenging problem which requires further improvements [12].

Presently, most EEG-based BCI systems are synchronous, which means the user can only use the BCI during imposed time windows [12]. An ideal BCI should be self-paced (asynchronous), to allow the user to use it at any time [5]. However, designing a selfpaced $\mathrm{BCI}$ is a challenging problem which requires continous analysis of EEG signals. This analysis should determine if the user is in an Intentional Control (IC) state, i.e., if he is producing one of the brain activity patterns used to control the BCI, or, conversely, if he is in a Non Control (NC) state [5]. Finally, if the user is in an IC state, the system must also determine which kind of brain activity pattern is being produced [5].

In this paper, we consider the design of a self-paced $\mathrm{BCI}$ as a pattern rejection problem [8], where NC states must be rejected by the BCI, whereas IC states must be accepted and properly classified. In the BCI literature, two main strategies are employed to deal with the NC state: the use of thresholds on reliability functions (TRF reject option) or the use of specialized classifiers (SC reject option). Algorithms of the first category use one or two thresholds, generally manually defined, on reliability functions $[6,11]$. If the reliability function, here the classifier output, is higher than the given threshold, then the IC state is chosen, otherwise rejection is performed and the NC state is chosen. In the second category, specialized classifiers, known as reject classifiers, are used to distinguish IC from NC states [10, 2, 13]. Another classifier, known as the recognition classifier, is then used to distinguish between the targeted patterns. Generally, a different set of features is used for the reject classifiers and for the recognition classifier.

So far, despite the need to design efficient self-paced $\mathrm{BCI}$, relatively few algorithms have been explored to deal with the NC state. Moreover, to our best knowledge, no study has systematically compared several reject options using several classifiers in the field of BCI. This lack of study prevents from identifying the desirable properties of reject options and classifiers for selfpaced BCI design. In this paper, we first introduce two reject options that have not been used yet in the BCI field: the rejection class strategy and TRF based on the automatic multi-threshold learning algorithm. Second, we assess and compare several reject options using several kinds of classifiers in order to study their behavior and identify the most appropriate ones. 


\section{Method}

In this Section, we present the different classifiers and reject options that we investigated.

\subsection{Classifiers}

For this study we used four different classifiers, which exhibit different properties with regards to classification performance and rejection. Two of these classifiers are generative classifiers and two are discriminant classifiers. The generative classifiers describe the training data, which is interesting to reject the $\mathrm{NC}$ state using reliability functions and to generalize using noisy training data. The discriminant classifiers have powerful classification performance. As classifiers, we used:

- A Support Vector Machine (SVM): SVM are discriminant classifiers, efficient for BCI design [3]. We used Gaussian kernel to obain nonlinear SVM.

- A Radial Basis Function Network (RBFN): RBFN are neural networks using radial basis functions learned using non supervised clustering. Thus, RBFN are generative through their hidden layer and discriminant through their output layer.

- A Fuzzy Inference System (FIS): FIS are a set of fuzzy "if-then" rules. FIS are nonlinear and generative classifiers with numerous advantages for BCI design [4].

- A Linear Discriminant Analysis (LDA) classifier: LDA are linear and discriminant classifiers widely used for BCI purposes. They use hyperplanes to separate classes [3].

All classifiers have been trained on the same training data set (see section 3.1). The meta parameters of each classifier have been optimized for each data set separately, by splitting the training data into a training set and a validation set.

\subsection{Reject options}

The NC rejection task is an outlier rejection problem [8]. Thus, data from NC states are outliers and data from IC states are target class data. We compared three reject options: SC, TRF and rejection class.

\subsubsection{Specialized classifier (SC)}

A specialized two-class classifier is trained independently from the target classifier to reject - or not - the input pattern. Separating the recognition and the rejection classifier allows the rejection classifier to take advantage of another family of classifiers or a different set of features.

\subsubsection{Rejection class (RC)}

A rejection class is added to the recognition problem [7]. Outliers are treated as the other target classes. To our best knowledge, this method has not been considered yet for BCI purposes.

\subsubsection{Thresholds on reliability functions (TRF)}

TRF uses the knowledge of the recognition classifier through reliability functions [8]. TRF use the interpretation of reliability functions: the lower is the confidence (i.e., the reliability function value), the more the pattern must be rejected. Thus, the TRF reject option is defined with a set of $N$ thresholds each one associated with a reliability function. If all functions are lower than their respective thresholds, rejection is performed. The main problem is to set the threshold values which is increasingly difficult as the number of thresholds increases. Interestingly enough, most self-paced BCI based on TRF use a single threshold, manually defined. In this paper, we introduce the use of the Automatic Multiple-Threshold Learning algorithm (AMTL), developed by Mouchere and Anquetil, in the BCI field [7, 8]. AMTL is a generic greedy algorithm based on empiric heuristics. We used two variants AMTL1 and AMTL2 with different aims. AMTL1 finds the best trade-off between the rejection of the data from the target classes and the rejection of outliers. AMTL2 finds the best description of target classes without using outliers. We can note that TRF include classical approaches which only use the score of the best class to make the reject decision. In the following, we denote as AMTL-MT, TRF using Multiple Thresholds, and as AMTL-ST, TRF using a Single Threshold on the best class score.

The SC and RC reject options should take advantage of discriminant classifiers because they consider the rejection problem as a simple classification task. The TRF architecture needs reliability functions representing generative knowledge for the rejection of the NC state [7]. We used the classifier output scores as reliability functions. This is relevant for RBFN and FIS but less relevant for SVM and LDA. With AMTL2, we used the activation of radial basis functions for RBFN to have a better target class description. 


\section{Evaluation}

The previously mentioned methods were evaluated on motor imagery data, using Receiver Operating Characteristic (ROC) analysis and accuracy computation.

\subsection{Motor imagery EEG data used}

Evaluations were achieved on 4 EEG data sets of Motor Imagery (MI) acquired from 2 healthy subjects, beginners with BCI. During the experiments, subjects were asked to perform MI, i.e., imagination of left or right hand movements [9]. For each subject, data were collected over 2 days during which 3 to 5 sessions were recorded each day. A session was composed of 20 trials of each of the two classes (LEFT or RIGHT), arranged in a random order. A trial lasted 8 seconds, during which the subject received instructions the first 3 seconds and had to perform the required MI task during the last 5 seconds. We specifically asked subjects not to perform MI nor real movements outside these 5 second periods dedicated to MI.

EEG signals were sampled at $512 \mathrm{~Hz}$ and recorded using electrodes FC3, FC4, C5, C3, C1, C2, C4, C6, $\mathrm{CP} 3, \mathrm{CP} 4$, with nose reference. These electrodes cover the motor cortex area, and are standard electrode positions, placed according to the extended 10-20 system [1]. It should also be noted that a sampling frequency of $512 \mathrm{~Hz}$ is suitable for our problem, as the relevant EEG power variations triggered by MI are in the 8-30 $\mathrm{Hz}$ frequency band [9]. For each subject and each day, the first half of the sessions was used to build a training set whereas the remaining sessions were used to build a test set. Hence, we used a total of 4 data sets, each one being composed of a training set and a test set. EEG signals from the training sets were visually inspected and periods of MI polluted by artifacts were removed. No artifact was removed from the test sets.

\subsection{Data labelling}

We labelled as belonging to the LEFT or RIGHT class the samples that were in the MI period of each trial, according to the imagined movement the subject was asked to perform. Samples from the first $0.5 \mathrm{~s}$ of each MI period were labelled as NC in order to take into account the user's reaction time. All other samples were also labelled as belonging to the NC state. Then, EEG signals were segmented into $1 \mathrm{~s}$ segments with $93.75 \%(15 / 16)$ of overlap between consecutive segments. Each segment was labelled according to the most represented label among the samples composing it. Then, a feature vector was extracted from each segment and labelled with this segment label. As such, 16 feature vectors were extracted for each second.

\subsection{Preprocessing and feature extraction}

In order to build the classifier inputs, we applied temporal and spatial filters to EEG signals and extracted Band Power (BP) features from these signals. More specifically, EEG signals were first band-pass filtered in 3-45 Hz. Then, from the 10 initial EEG channels, 2 new channels were designed by applying a discrete surface Laplacian spatial filter over channel C3 and C4 [12]. Finally, we extracted logarithmic BP features from these two Laplacian channels [9]. Computing a BP feature consists in band-pass filtering the signal in a given frequency band, squaring it, averaging it over the whole time segment and taking its logarithm. BP features are popular features which are known to be efficient for MI classification [9]. Indeed, imagination of hand movements is known to trigger EEG power variations, mainly in the $\mu(\simeq 8-13 \mathrm{~Hz})$ and $\beta(\simeq 13-30 \mathrm{~Hz})$ frequency bands, over the motor cortex areas [9].

Two sets of BP features were generated: features for rejection and features for recognition. The first set was obtained by extracting BP features in the frequency bands that best differentiate IC from NC, whereas the second set was obtained using frequency bands that best differentiate left MI from right MI. For each subject, these frequency bands were identified using a statistical analysis in a way similar to [4]. This analysis compared the BP mean value for the two corresponding conditions (NC versus IC or left MI versus right MI) for different frequencies in the $4-35 \mathrm{~Hz}$ frequency band, with the aim of selecting the most discriminative frequency bands. Features for rejection were used as input of the reject classifiers whereas features for recognition were used for the recognition classifiers.

\subsection{Results}

In order to evaluate how well the classifiers and reject options can reject the NC state, we conducted a ROC analysis [5]. The ROC curve allows to consider the trade-off between the True Acceptance Rate (TAR) and the False Acceptance Rate (FAR) for a reject option. FAR and TAR are based on the number of True Positive (TP, acceptance of an IC state), of True Negative (TN, rejection on an NC state), of False Positive (FP, acceptance of an NC state) and of False Negative (FN, rejection of an IC state) and are defined as follows:

$$
T A R=\text { Recall }=\frac{T P}{T P+F N}
$$




$$
F A R=\frac{F P}{F P+T N}
$$

FAR and TAR represent the rejection performances of the evaluated system as they are independent of the proportion between IC and NC states. As an evaluation measure, we also considered the Precision:

$$
\text { Precision }=\frac{T P}{T P+F P}
$$

Precision is linked to the comfort of the final user, as it summarized how often the BCI system will respond correctly. Precision depends on the proportion between IC and NC states. Then, to evaluate the classification capabilities of the methods, we computed the accuracy of each method [5] for a fixed FAR. The accuracy is defined as the percentage of accepted IC state that have been correctly classified.

Table 1 displays the Area Under the ROC Curve (AUC), for FAR lower than or equal to 0.2, obtained by all methods. We chose to use the AUC for FAR $\leq 0.2$ rather than the total AUC, as Mason et al highlighted that only the beginning of the ROC curves was relevant for BCI [5]. Actually, a high FAR tends to cause excessive user frustration making the resulting BCI not usable. Here, it should be noted that the AUC for FAR $\leq$ 0.2 would be 0.02 for a randomly performing classifier.

We computed the accuracy and precision of each classifier and reject option, averaged over the four data sets for a fixed FAR of $10 \%$. This FAR is similar to the FAR used in the work of Scherer et al [10]. Table 2 displays the resulting accuracy, precision and TAR.

Results showed that using a nonlinear classifier within the RC reject option led to the most efficient selfpaced BCI. Independently from the reject option used, nonlinear classifiers, i.e., FIS, RBFN or SVM, provided the best rejection results. Using TRF, LDA provided the highest accuracy, but this has to be moderated by the low TAR it provided. Actually, it is very likely that LDA was in fact performing ambiguity rejection [8] and not outlier rejection, which could explain the results.

Concerning the reject options, the obtained AUC and TAR may appear as modest, but it should be noted that they are in line with results found in the literature. For instance, the 3-class self-paced BCI presented in the work of Scherer et al obtained an averaged FAR of 16.9 $\%$ and an average TAR of $28.4 \%$ [10]. Moreover, it is known that designing an EEG-based self-paced BCI is a difficult problem which requires further research $[3,5,10]$.

The most efficient methods in terms of rejection capabilities are RC and SC. However, RC outperformed
Table 1. Rejection capabilities: area under the $R O C$ curves for $F A R \leq \mathbf{0 . 2}$, for all data

\begin{tabular}{|c|c|c|c|c|c|}
\hline \multirow{2}{*}{$\begin{array}{l}\text { reject } \\
\text { option }\end{array}$} & \multirow[t]{2}{*}{ Classifier } & \multicolumn{2}{|c|}{ Subject 1} & \multicolumn{2}{|c|}{ Subject 2} \\
\hline & & Day1 & Day 2 & Day 1 & Day 2 \\
\hline \multirow{4}{*}{$\mathrm{SC}$} & SVM & 0.105 & 0.077 & 0.057 & 0.046 \\
\hline & FIS & 0.102 & 0.075 & 0.052 & 0.039 \\
\hline & RBFN & 0.103 & 0.074 & 0.055 & 0.044 \\
\hline & LDA & 0.102 & 0.071 & 0.041 & 0.035 \\
\hline \multirow{4}{*}{$\mathrm{RC}$} & SVM & 0.102 & 0.077 & 0.056 & $\overline{0.062}$ \\
\hline & FIS & 0.102 & 0.072 & 0.055 & 0.052 \\
\hline & $\overline{\text { RBFN }}$ & 0.095 & 0.075 & 0.054 & 0.058 \\
\hline & LDA & 0.095 & 0.072 & 0.053 & 0.048 \\
\hline \multirow{4}{*}{$\begin{array}{c}\text { TRF } \\
\text { AMTL1 } \\
\text { ST }\end{array}$} & $\overline{\text { SVM }}$ & 0.025 & $\overline{0.040}$ & 0.028 & $\overline{0.033}$ \\
\hline & FIS & 0.057 & 0.039 & 0.04 & 0.036 \\
\hline & RBFN & 0.053 & 0.043 & 0.033 & 0.026 \\
\hline & LDA & 0.02 & 0.036 & 0.047 & 0.036 \\
\hline \multirow{4}{*}{$\begin{array}{c}\text { TRF } \\
\text { AMTL1 } \\
\text { MT }\end{array}$} & SVM & 0.025 & 0.041 & 0.028 & 0.032 \\
\hline & FIS & 0.082 & 0.06 & 0.037 & 0.042 \\
\hline & RBFN & 0.066 & 0.047 & 0.030 & 0.028 \\
\hline & LDA & 0.038 & 0.039 & 0.038 & 0.037 \\
\hline \multirow{4}{*}{$\begin{array}{c}\text { TRF } \\
\text { AMTL2 } \\
\text { MT }\end{array}$} & SVM & 0.025 & 0.040 & 0.028 & $\overline{0.032}$ \\
\hline & FIS & 0.058 & 0.044 & 0.041 & 0.042 \\
\hline & RBFN & 0.065 & $\overline{0.050}$ & 0.030 & 0.028 \\
\hline & LDA & 0.021 & 0.027 & 0.039 & 0.035 \\
\hline
\end{tabular}
sets and methods.

SC in terms of accuracy for a fixed FAR of $10 \%$. TRF had the lowest rejection capabilities, even if with a low resource cost the use of multiple thresholds improved the results as compared to a single threshold, especially for generative classifiers. Indeed, regarding the AUC in Table 1, it can be noticed that discriminant classifiers, i.e., SVM and LDA, obtained scores that are close to random classification scores with TRF. However, it is interesting to note that TRF provided the highest accuracy. This suggests that, implicitely, TRF also performed ambiguity rejection in addition to outlier rejection. In the future, it could be interesting to incorporate and study ambiguity rejection in our BCI in order to increase the accuracy.

\section{Conclusion}

This paper first introduced two pattern rejection strategies for self-paced BCI design: the RC reject option and the TRF reject option based on the AMTL algorithm. Then, it compared the SC, RC and TRF 
Table 2. Classification capabilities: average Accuracy (Acc), TAR and Precison (Prec), in percent, for a fixed FAR of $10 \%$.

\begin{tabular}{|c|l|l|l|l|l|}
\hline & & SVM & FIS & RBFN & LDA \\
\hline \multirow{4}{*}{ SC } & Acc & $74.1 \pm 8$ & $73.2 \pm 5.2$ & $73,9 \pm 9$ & $72.0 \pm 4.7$ \\
\cline { 2 - 6 } & TAR & $38.2 \pm 15.2$ & $34.3 \pm 16.6$ & $37.0 \pm 15$ & $33.1 \pm 17.7$ \\
\cline { 2 - 6 } & Prec & $69.1 \pm 8.3$ & $65.7 \pm 11.6$ & $68.3 \pm 8.8$ & $64.6 \pm 12.1$ \\
\hline \hline \multirow{4}{*}{ RC } & Acc & $83.4 \pm 7.7$ & $79.4 \pm 7.3$ & $80.2 \pm 8.3$ & $81.1 \pm 7.3$ \\
\cline { 2 - 6 } & TAR & $\mathbf{4 0 . 0} \pm \mathbf{1 2 . 2}$ & $38.7 \pm 15.2$ & $38.2 \pm 10.5$ & $36.1 \pm 12.3$ \\
\cline { 2 - 6 } & Prec & $\mathbf{7 0 . 8} \pm \mathbf{6 . 2}$ & $69.5 \pm 7.8$ & $70.0 \pm 5.8$ & $68.4 \pm 7.4$ \\
\hline \hline \multirow{3}{*}{$\begin{array}{c}\text { AMTL1 } \\
\text { ST }\end{array}$} & Acc & $84.1 \pm 5.7$ & $92.6 \pm 7.1$ & $82.7 \pm 9.1$ & $\mathbf{9 4 . 5} \pm \mathbf{5}$ \\
\cline { 2 - 6 } & TAR & $16.3 \pm 3.6$ & $22.8 \pm 4.9$ & $20.1 \pm 6.4$ & $17.2 \pm 8.8$ \\
\cline { 2 - 6 } & Prec & $50.5 \pm 5.5$ & $58.7 \pm 5$ & $55.0 \pm 8.3$ & $48.9 \pm 16.5$ \\
\hline \hline \multirow{3}{*}{ AMTL1 } & Acc & $84.1 \pm 5.8$ & $77.6 \pm 8.1$ & $83.5 \pm 8.1$ & $93.2 \pm 4.8$ \\
\cline { 2 - 6 } & TAR & $16.2 \pm 3.4$ & $28.5 \pm 11.1$ & $22.2 \pm 6.5$ & $19.3 \pm 2.2$ \\
\cline { 2 - 6 } & Prec & $50.4 \pm 5.3$ & $62.8 \pm 8.8$ & $57.6 \pm 7.4$ & $55.0 \pm 3.2$ \\
\hline \hline \multirow{2}{*}{ AMTL2 } & Acc & $83.8 \pm 5.8$ & $92.1 \pm 6.4$ & $75.9 \pm 6.6$ & $94.1 \pm 3.9$ \\
\cline { 2 - 6 } MT & TAR & $16.2 \pm 3.6$ & $24.1 \pm 4.7$ & $22.5 \pm 9.8$ & $13.6 \pm 6.3$ \\
\cline { 2 - 6 } & Prec & $50.4 \pm 5.5$ & $60.1 \pm 4.7$ & $56.8 \pm 11.1$ & $44.3 \pm 12.9$ \\
\hline
\end{tabular}

reject options using SVM, FIS, RBFN and LDA classifiers, on motor imagery data. The results showed that Gaussian SVM, FIS and RBFN classifiers reached the best rejection performance and that RC outperformed SC which outperformed TRF. To conclude, we could recommend using the $\mathrm{RC}$ reject option with nonlinear classifiers for efficient self-paced BCI design.

Acknowledgements: This work was partially supported by the French National Research Agency within the OpenViBE project (grant ANR05RNTL01601). The authors would also like to thank Morgane Rosendale for her helpful comments on the manuscript.

\section{References}

[1] American electroencephalographic society: Guidelines for standard electrode position nomenclature. J Clin Neurophysiol., 8(2):200-202, 1991.

[2] A. Bashashati, R. Ward, and G. Birch. Towards development of a 3-state self-paced brain-computer interface. Comput. Intell. Neurosci., 2007.

[3] F. Lotte, M. Congedo, A. Lécuyer, F. Lamarche, and B. Arnaldi. A review of classification algorithms for eeg-based brain-computer interfaces. Journal of Neural Engineering, 4:R1-R13, 2007.

[4] F. Lotte, A. Lécuyer, F. Lamarche, and B. Arnaldi. Studying the use of fuzzy inference systems for motor imagery classification. IEEE Trans. on Neural Sys. and Rehab., 15(2):322-324, 2007.
[5] S. Mason, J. Kronegg, J. Huggins, M. Fatourechi, and A. Schloegl. Evaluating the performance of selfpaced bci technology. Technical report, http://www.bciinfo.tugraz.at/Research_Info/documents/articles, 2006.

[6] J. Millán and J. Mouriño. Asynchronous BCI and local neural classifiers: An overview of the Adaptive Brain Interface project. IEEE Trans. on Neural Sys. and Rehab., 2003.

[7] H. Mouchère and E. Anquetil. Generalization capacity of handwritten outlier symbols rejection with neural network. In Proc. of IWFHR'06, pages 187-192, 2006.

[8] H. Mouchère and E. Anquetil. A unified strategy to deal with different natures of reject. In Proc. of ICPR'06, pages 792-795, 2006.

[9] G. Pfurtscheller and C. Neuper. Motor imagery and direct brain-computer communication. Proceedings of the IEEE, 89(7):1123-1134, 2001.

[10] R. Scherer, F. Lee, A. Schlögl, R. Leeb, H. Bischof, and G. Pfurtscheller. Towards self-paced brain-computer communication: Navigation through virtual worlds. IEEE, Trans. on Biomed. Eng., 55(2):675-682, 2008.

[11] G. Townsend, B. Graimann, and G. Pfurtscheller. Continuous eeg classification during motor imagerysimulation of an asynchronous bci. IEEE Trans. on Neural Sys. and Rehab., 12(2):258-265, 2004.

[12] J. Wolpaw, N. Birbaumer, D. McFarland, G. Pfurtscheller, and T. Vaughan. Brain-computer interfaces for communication and control. Clinical Neurophysiology, 113(6):767-791, 2002.

[13] D. Zhang, Y. Wang, X. Gao, B. Hong, and S. Gao. An algorithm for idle-state detection in motor-imagerybased brain-computer interface. Comput. Intell. Neurosci., 2007. 